\title{
Output Feedback Control for Couple-Group Consensus of Multiagent Systems
}

\author{
Huanyu Zhao, Hongbiao Zhou, and Zhongyi Tang \\ Faculty of Electronic and Electrical Engineering, Huaiyin Institute of Technology, Huaian, Jiangsu 223003, China \\ Correspondence should be addressed to Huanyu Zhao; hyzhao10@gmail.com
}

Received 18 January 2014; Accepted 5 April 2014; Published 24 April 2014

Academic Editor: Hao Shen

Copyright ( 2014 Huanyu Zhao et al. This is an open access article distributed under the Creative Commons Attribution License, which permits unrestricted use, distribution, and reproduction in any medium, provided the original work is properly cited.

\begin{abstract}
This paper deals with the couple-group consensus problem for multiagent systems via output feedback control. Both continuousand discrete-time cases are considered. The consensus problems are converted into the stability problem of the error systems by the system transformation. We obtain two necessary and sufficient conditions of couple-group consensus in different forms for each case. Two different algorithms are used to design the control gains for continuous- and discrete-time case, respectively. Finally, simulation examples are given to show the effectiveness of the proposed results.
\end{abstract}

\section{Introduction}

During the past decade, consensus problem of multiagent system has attracted a lot of attentions in control area [111]. It is mainly due to its wide applications in practice, such as sensor networks, unmanned aerial vehicles, and robotics. In [2], the authors studied the consensus seeking problem of multiagent systems with dynamically changing interaction topologies, where both discrete and continuous consensus algorithms were considered. In [9], the authors studied the containment problem of linear multiagent systems, where a pinning control strategy was designed for a part of agents such that all the agents can achieve a consensus with the leader asymptotically. A second-order consensus problem for multiagent systems with nonlinear dynamics and directed topologies was studied in [10]. More works about consensus problem were surveyed in [11].

Sometimes the interaction topology does not have a spanning tree, while it contains two or more subgraphs which include a spanning tree, respectively. In this case, some researchers studied the group consensus problem [12-15]. In [12], the authors studied the group consensus problem of multiagent systems with switching topologies. The group consensus was proved to be equivalent to the asymptotical stability of a class of switched linear systems by a double-treeform transformation. In [13], two different kinds of consensus protocols were given to deal with the group consensus problem for double-integrator dynamic multiagent systems. In [15], the sampled-data control method was employed to deal with the group consensus problem for multiagent systems, where the interaction topology is undirected.

Sometimes the system states are not known completely, while the output of the systems is measurable. The output will be used to design the controller for this case, that is, output feedback controller. Recently, the output feedback control problems have been reported in a lot of literature [1619]. In [16], the output feedback robust stabilization problem for a class of jump linear system was studied. In [17], the authors studied the finite-time stabilization of continuoustime linear systems via dynamic output feedback. In [18], the Lyapunov-Metzler inequalities were used to study the dynamic output feedback control problem of switched linear systems. Very recently, the method based on output feedback control has been used to analyze the networked systems [2023]. In [20], the consensusability of a class of linear multiagent systems was studied, where the agent updates its information by using the neighbor's output. In [21], the output regulation theory was used to study the output consensus problems for heterogeneous uncertain linear multiagent systems. In [22], by using appropriate coordinate transformation, a new consensus algorithm via dynamic output feedback control for multiagent systems was studied. While in [23], the joint 
effects of agent dynamic and network topology on the consensusability of linear discrete-time multiagent systems via relative output feedback were studied.

Motivated by the aforementioned works, we will investigate the couple-group consensus problems for multiagent systems via output feedback control. The systems considered include both continuous-time case and discrete-time case. We convert the couple-group consensus problems of multiagent systems into the stability problems of the error systems by a system transformation. Based on linear system theory, some necessary and sufficient conditions for couple-group consensus are obtained. For continuous-time case, the algorithm based on homotopy method is given to compute the allowable control gain. For discrete-time case, the algorithm based on cone complementary linearization method is given to compute the allowable control gain.

Notation. Let $\mathbb{R}$ and $\mathbb{N}$ represent, respectively, the real number set and the nonnegative integer set. Denote the spectral radius of the matrix $M$ by $\rho(M)$. Suppose that $A, B \in \mathbb{R}^{p \times p}$. Let $A \geq B$ (resp., $A>B$ ) denote that $A-B$ is symmetric positive semidefinite (resp., symmetric positive definite). $I_{n}$ denotes the $n \times n$ identity matrix. $\operatorname{Re}(\cdot)$ and $\operatorname{Im}(\cdot)$ represent, respectively, the real part and imaginary part of a number. Let $\mathbf{0}$ denote zero matrix with appropriate dimensions.

\section{Preliminaries and Problem Formulation}

Graph Theory. Let $\mathscr{G}=(\mathscr{V}, \mathscr{E}, \mathscr{A})$ be a directed graph of order $n$, where $\mathscr{V}=\left\{v_{1}, \ldots, v_{n}\right\}$ and $\mathscr{E}$ represent the node set and the edge set, respectively. $\mathscr{A}=\left[a_{i j}\right] \in \mathbb{R}^{n \times n}$ is the adjacency matrix associated with $\mathscr{G}$, where $a_{i j}>0$ if $\left(v_{i}, v_{j}\right) \in \mathscr{E}$, otherwise, $a_{i j}=0$. An edge $\left(v_{i}, v_{j}\right) \in \mathscr{E}$ if agent $j$ can obtain the information from agent $i$. We say agent $i$ is a neighbor of agent $j$. Let $N_{i}=\left\{v_{j} \in \mathscr{V}:\left(v_{i}, v_{j}\right) \in \mathscr{E}\right\}$ denote the neighbor set of agent $i$. The (nonsymmetrical) Laplacian matrix $\mathscr{L}$ associated with $\mathscr{A}$ and hence $\mathscr{G}$ is defined as $\mathscr{L}=\left[l_{i j}\right] \in \mathbb{R}^{n \times n}$, where $l_{i i}=\sum_{j=1, j \neq i}^{n} a_{i j}$ and $l_{i j}=-a_{i j}$, for all $i \neq j$. A directed path is a sequence of edges in a directed graph in the form of $\left(v_{i_{1}}, v_{i_{2}}\right),\left(v_{i_{2}}, v_{i_{3}}\right), \ldots$, where $v_{i_{k}} \in \mathscr{V}$. A directed tree is a directed graph, where every node has exactly one parent except for one node, called the root, which has no parent, and the root has a directed path to every other node. A directed spanning tree of $\mathscr{G}$ is a directed tree that contains all nodes of $\mathscr{G}$. A directed graph has or contains a directed spanning tree if there exists a directed spanning tree as a subset of the directed graph; that is, there exists at least one node having a directed path to all of the other nodes.

Suppose that the multiagent systems considered consist of $n+m$ agents. In this paper, we will consider both continuoustime case and discrete-time case. We assume that the first $n$ agents achieve a consistent state while the last $m$ agents achieve another consistent state. Let $\mathscr{G}=(\mathscr{V}, \mathscr{E}, \mathscr{A})$ denote the topology of multiagent system considered. Denote $\mathscr{I}_{1}=$ $\{1,2, \ldots, n\}, \mathscr{I}_{2}=\{n+1, n+2, \ldots, n+m\}$. Let $\mathscr{V}_{1}=\left\{v_{1}, v_{2}\right.$, $\left.\ldots, v_{n}\right\}$ and $\mathscr{V}_{2}=\left\{v_{n+1}, v_{n+2}, \ldots, v_{n+m}\right\}$ represent the first $n$ agents and the last $m$ agents, respectively. Then, $\mathscr{V}=\mathscr{V}_{1} \cup \mathscr{V}_{2}$, $\mathscr{V}_{1} \cap \mathscr{V}_{2}=\Phi$. In addition, let $N_{1 i}=\left\{v_{j} \in \mathscr{V}_{1}:\left(v_{i}, v_{j}\right) \in \mathscr{E}\right\}$ and $N_{2 i}=\left\{v_{j} \in \mathscr{V}_{2}:\left(v_{i}, v_{j}\right) \in \mathscr{E}\right\}$.
For continuous-time case, the $i$ th agent's dynamics are as follows:

$$
\begin{gathered}
\dot{x}_{i}(t)=A x_{i}(t)+B u_{i}(t), \\
y_{i}(t)=C x_{i}(t), \\
i=1, \ldots, n+m,
\end{gathered}
$$

where $x_{i}(t) \in \mathbb{R}$ is the state, $u_{i}(t) \in \mathbb{R}$ is the control input, and $y_{i}(t) \in \mathbb{R}$ is the output. $A, B, C \in \mathbb{R}$ are the system coefficients.

For discrete-time case, the $i$ th agent's dynamics are as follows:

$$
\begin{gathered}
x_{i}[k+1]=A x_{i}[k]+B u_{i}[k], \\
y_{i}[k]=C x_{i}[k], \\
i=1, \ldots, n+m,
\end{gathered}
$$

where $x_{i}[k] \in \mathbb{R}$ is the state, $u_{i}[k] \in \mathbb{R}$ is the control input, and $y_{i}[k] \in \mathbb{R}$ is the output. $A, B, C \in \mathbb{R}$ are the system coefficients.

Sometimes the agent's state is difficult to obtain, while the output is measurable. Our main purpose in this paper is to design consensus algorithm based on the output such that the multiagent systems can achieve couple-group consensus. We consider the following consensus algorithms for continuoustime case and discrete-time case, respectively.

Continuous-Time Case. Consider

$$
\begin{aligned}
& u_{i}(t) \\
& \alpha\left[\sum_{j \in N_{1 i}} a_{i j}\left(y_{j}(t)-y_{i}(t)\right)+\sum_{j \in N_{2 i}} a_{i j} y_{j}(t)\right] \quad \forall i \in \mathscr{I}_{1}, \\
& \alpha\left[\sum_{j \in N_{1 i}} a_{i j} y_{j}(t)+\sum_{j \in N_{2 i}} a_{i j}\left(y_{j}(t)-y_{i}(t)\right)\right] \quad \forall i \in \mathscr{I}_{2},
\end{aligned}
$$

where $a_{i j} \geq 0$ for all $i, j \in \mathscr{I}_{1}, a_{i j} \geq 0$ for all $i, j \in \mathscr{I}_{2}$, and $a_{i j} \in \mathbb{R}$ for all $\left(v_{i}, v_{j}\right) \in \mathscr{E}_{o}=\left\{(i, j): i \in \mathscr{I}_{1}, j \in \mathscr{I}_{2}\right\} \cup\{(i, j):$ $\left.j \in \mathscr{I}_{1}, i \in \mathscr{I}_{2}\right\} . \alpha$ is the control gain to be designed.

\section{Discrete-Time Case. Consider}

$u_{i}[k]$

$$
=\left\{\begin{array}{l}
\gamma\left[\sum_{j \in N_{1 i}} a_{i j}\left(y_{j}[k]-y_{i}[k]\right)+\sum_{j \in N_{2 i}} a_{i j} y_{j}[k]\right] \\
\gamma\left[\sum_{j \in N_{1 i}} a_{i j} y_{j}[k]+\sum_{j \in N_{2 i}} a_{i j}\left(y_{j}[k]-y_{i}[k]\right)\right] \\
\forall i \in \mathscr{I}_{1},
\end{array},\right.
$$

where $a_{i j} \geq 0$ for all $i, j \in \mathscr{I}_{1}, a_{i j} \geq 0$ for all $i, j \in \mathscr{I}_{2}$, and $a_{i j} \in \mathbb{R}$ for all $\left(v_{i}, v_{j}\right) \in \mathscr{E}_{o}=\left\{(i, j): i \in \mathscr{I}_{1}, j \in \mathscr{I}_{2}\right\} \cup$ $\left\{(i, j): j \in \mathscr{I}_{1}, i \in \mathscr{I}_{2}\right\} \cdot \gamma$ is the control gain to be designed. In addition, we suppose the algorithms in (3) and (4) satisfy similar assumption to that of [24].

Assumption 1. (1): $\sum_{j=n+1}^{n+m} a_{i j}=0$ for all $i \in \mathscr{I}_{1} ;(2): \sum_{j=1}^{n} a_{i j}=$ 0 for all $i \in \mathscr{I}_{2}$. 
Assumption 2. The subgraphs $\mathscr{G}_{1}$ and $\mathscr{G}_{2}$ have a directed spanning tree, respectively. yields

Denote $X(t) \triangleq\left[x_{1}(t), \ldots, x_{n+m}(t)\right]^{T}$. Using (3) in (1)

$$
\dot{X}(t)=\left[\begin{array}{cc}
A I_{n}-\alpha B C \mathscr{L}_{1} & \alpha B C \Omega_{1} \\
\alpha B C \Omega_{2} & A I_{m}-\alpha B C \mathscr{L}_{2}
\end{array}\right] X(t),
$$

where $\mathscr{L}_{1}$ and $\mathscr{L}_{2}$ are the Laplacian matrices corresponding to subgraphs $\mathscr{G}_{1}$ and $\mathscr{G}_{2}$, respectively, and

$$
\begin{aligned}
& \Omega_{1}=\left[\begin{array}{cccc}
a_{1(n+1)} & a_{1(n+2)} & \cdots & a_{1(n+m)} \\
\vdots & \vdots & \cdots & \vdots \\
a_{n(n+1)} & a_{n(n+2)} & \cdots & a_{n(n+m)}
\end{array}\right], \\
& \Omega_{2}=\left[\begin{array}{cccc}
a_{(n+1) 1} & a_{(n+1) 2} & \cdots & a_{(n+1) n} \\
\vdots & \vdots & \cdots & \vdots \\
a_{(n+m) 1} & a_{(n+m) 2} & \cdots & a_{(n+m) n}
\end{array}\right] .
\end{aligned}
$$
yields

Denote $X[k] \triangleq\left[x_{1}[k], \ldots, x_{n+m}[k]\right]^{T}$. Using (4) in (2)

$$
X[k+1]=\left[\begin{array}{cc}
A I_{n}-\alpha B C \mathscr{L}_{1} & \alpha B C \Omega_{1} \\
\alpha B C \Omega_{2} & A I_{m}-\alpha B C \mathscr{L}_{2}
\end{array}\right] X[k],
$$

where $\mathscr{L}_{1}, \mathscr{L}_{2}, \Omega_{1}$, and $\Omega_{2}$ are the same as that of continuoustime case.

Remark 3. The group consensus problem of continuous time multiagent systems was studied in $[12,13,15]$. In [14], the authors studied the group consensus problem for discretetime multiagent systems. However, the couple-group consensus problem for the multiagent systems with stochastic switching topologies has not been researched. In addition, our method in this paper is based on the output feedback control, which is different from the existing results.

Our main purpose is to give the conditions for couplegroup consensus. We next convert the consensus problem of multiagent system into the stability problem of the error systems. Before giving the main results, the following definitions and lemma are needed.

Definition 4 (see [24]). The multiagent system in (5) is said to achieve couple-group consensus if the states of agents satisfy (i) $\lim _{t \rightarrow \infty}\left\|x_{i}(t)-x_{j}(t)\right\|=0$, for all $i, j \in \mathscr{I}_{1}$ and (ii) $\lim _{t \rightarrow \infty}\left\|x_{i}(t)-x_{j}(t)\right\|=0$, for all $i, j \in \mathscr{I}_{2}$.

Definition 5 (see [24]). The multiagent system in (7) is said to achieve couple-group consensus if the states of agents satisfy (i) $\lim _{k \rightarrow \infty}\left\|x_{i}[k]-x_{j}[k]\right\|=0$, for all $i, j \in \mathscr{I}_{1}$ and (ii) $\lim _{k \rightarrow \infty}\left\|x_{i}[k]-x_{j}[k]\right\|=0$, for all $i, j \in \mathscr{I}_{2}$.

Lemma 6 (see [25] Schur complements). Consider a hermitian matrix $Q$ such that $Q=\left[\begin{array}{ll}Q_{11} & Q_{12} \\ Q_{12}^{T} & Q_{22}\end{array}\right]$. Then, $Q>0$ if and only if

$$
\begin{gathered}
Q_{22}>0, \\
Q_{11}-Q_{12} Q_{22}^{-1} Q_{12}^{T}>0
\end{gathered}
$$

or

$$
\begin{gathered}
Q_{11}>0, \\
Q_{22}-Q_{12}^{T} Q_{11}^{-1} Q_{12}>0 .
\end{gathered}
$$

\section{Main Results}

In this section, we will give the main results of this paper.

\subsection{Continuous-Time Case. Let}

$$
\begin{aligned}
& z_{i}(t) \triangleq x_{i}(t)-x_{n}(t), \quad i=1, \ldots, n-1, \\
& z_{j}(t) \triangleq x_{j}(t)-x_{n+m}(t), \quad j=n+1, \ldots, n+m-1, \\
& Z(t) \triangleq\left[z_{1}(t), \ldots, z_{n-1}(t), z_{n+1}(t), \ldots, z_{n+m-1}(t)\right]^{T} .
\end{aligned}
$$

Then by some computations, we obtain the error systems as follows:

$$
\begin{aligned}
\dot{Z}(t) & =\left[\begin{array}{cc}
A I_{n-1}-\alpha B C \widetilde{\mathscr{L}}_{1} & \alpha B C \widetilde{\Omega}_{1} \\
\alpha B C \widetilde{\Omega}_{2} & A I_{m-1}-\alpha B C \widetilde{\mathscr{L}}_{2}
\end{array}\right] Z(t) \\
& \triangleq F_{c} Z(t) \\
& =\left(A I_{n+m-2}+\alpha B C \widetilde{F}_{c}\right) Z(t),
\end{aligned}
$$

where

$$
\begin{aligned}
& \widetilde{F}_{c}=\left[\begin{array}{cc}
-\widetilde{\mathscr{L}}_{1} & \widetilde{\Omega}_{1} \\
\widetilde{\Omega}_{2} & -\widetilde{\mathscr{L}}_{2}
\end{array}\right], \\
& \widetilde{\mathscr{L}}_{1}=\left[\begin{array}{ccc}
l_{11}-l_{n 1} & \cdots & l_{1(n-1)}-l_{n(n-1)} \\
\vdots & \cdots & \vdots \\
l_{(n-1) 1}-l_{n 1} & \cdots & l_{(n-1)(n-1)}-l_{n(n-1)}
\end{array}\right] \text {, } \\
& \widetilde{\mathscr{L}}_{2}=\left[\begin{array}{ccc}
l_{(n+1) 1}-l_{(n+m) 1} & \cdots & l_{(n+1)(n+m-1)}-l_{(n+m)(n+m-1)} \\
\vdots & \cdots & \vdots \\
l_{(n+m-1) 1}-l_{(n+m) 1} & \cdots & l_{(n+m-1)(n+m-1)}-l_{(n+m)(n+m-1)}
\end{array}\right] \text {, } \\
& \widetilde{\Omega}_{1}=\left[\begin{array}{ccc}
a_{1(n+1)}-a_{n(n+m)} & \cdots & a_{1(n+m-1)}-a_{n(n+m-1)} \\
\vdots & \cdots & \vdots \\
a_{(n-1)(n+1)}-a_{n(n+m)} & \cdots & a_{(n-1)(n+m-1)}-a_{n(n+m-1)}
\end{array}\right], \\
& \widetilde{\Omega}_{2}=\left[\begin{array}{ccc}
a_{(n+1) 1}-a_{(n+m) 1} & \cdots & a_{(n+1)(n-1)}-a_{(n+m)(n-1)} \\
\vdots & \cdots & \vdots \\
a_{(n+m-1) 1}-a_{(n+m) 1} & \cdots & a_{(n+m-1)(n-1)}-a_{(n+m)(n-1)}
\end{array}\right] .
\end{aligned}
$$

Here we have used Assumption 1 and the property of Laplacian matrix.

Now the couple-group consensus problem of (5) has been converted into the stability problem of error system (11). We next give our main results.

Theorem 7. The multiagent system (5) can achieve couplegroup consensus asymptotically if and only if $\alpha$ satisfies $A+$ $\alpha B C \operatorname{Re}\left(\mu_{i}\right)<0$, where $\mu_{i}(i=1, \ldots, n+m-2)$ is the ith eigenvalue of $\widetilde{F}_{c}$. 
Proof. According to the aforementioned discussion, we know that the multiagent systems (5) can achieve couple-group consensus asymptotically if and only if the error system (11) is asymptotically stable. It follows from linear system theory [26] that system (11) which is asymptotically stable is equivalent to all eigenvalues of $F_{c}$ having negative real parts. Denote the $i$ th eigenvalues of $F_{c}$ and $\widetilde{F}_{c}$, respectively, by $\eta_{i}$ and $\mu_{i}(i=1, \ldots, n+m-2)$. Then, $\eta_{i}=A+\alpha B C \mu_{i} . \operatorname{Re}\left(\eta_{i}\right)<0$ is equivalent to $A+\alpha B C \operatorname{Re}\left(\mu_{i}\right)<0$. This completes the proof.

Remark 8. Theorem 7 provides a necessary and sufficient condition of couple-group consensus for multiagent system (5). According to linear system theory, we know that system (11) which is asymptotically stable is equivalent to that in which there exists a positive matrix $P$ such that $P F_{c}+F_{c}^{T} P \prec$ 0 . Hence, we can get another condition of couple-group consensus for multiagent systems (5).

Theorem 9. The multiagent system (5) can achieve couplegroup consensus asymptotically if and only if there exists a positive definite matrix $P$ such that

$$
2 A P+\alpha B C\left(P \widetilde{F}_{c}+\widetilde{F}_{c}^{T} P\right) \prec 0
$$

holds.

Proof. The proof is straightforward; here is omitted.

Remark 10. Theorem 9 gives a necessary and sufficient condition for couple-group consensus in forms of matrix inequality. However, the matrix inequality in (13) is nonlinear with regard to variables $\alpha$ and $P$. Here we provide a numerical algorithm based on homotopy method to solve this problem. The similar method can be found in $[22,27,28]$.

\section{Algorithm 11. Consider the following}

Step 1. Introduce a real number $\lambda$ varying from 0 to 1 , and construct a matrix function

$$
H(\alpha, P, \lambda)=(1-\lambda) F_{1}(P)+\lambda F_{2}(\alpha, P)
$$

with $F_{1}(P)=2 A P, F_{2}(P)=\alpha B C\left(P \widetilde{F}_{c}+\widetilde{F}_{c}^{T} P\right)$.

Step 2 (Set $\lambda=0$ ). Compute the initial value of $P_{0}$ by solving the LMI $H(\alpha, P, 0) \prec \mathbf{0}$.

Step 3. Increase $\lambda$ by some homotopy path, such as $\lambda=(k / M)$ $(k=1,2, \ldots, M) . M$ is a large positive integer, for example, $M=1000$. Compute $\alpha^{0}$ by solving LMI $H\left(\alpha, P_{0}, 1 / M\right) \prec \mathbf{0}$.

Step 4 . Increase $\lambda$ by the same homotopy path as Step 3. Compute $P_{1}$ by solving LMI $H\left(\alpha^{0}, P, 2 / M\right) \prec \mathbf{0}$.

Step 5. Repeat Steps 3 and 4 until $\lambda$ reaches Step 1.

3.2. Discrete-Time Case. Similar to continuous-time case, we can get the similar results for discrete-time case.
Let

$$
\begin{aligned}
& z_{i}[k] \triangleq x_{i}[k]-x_{n}[k], \quad i=1, \ldots, n-1, \\
& z_{j}[k] \triangleq x_{j}[k]-x_{n+m}[k], \quad j=n+1, \ldots, n+m-1, \\
& Z[k] \triangleq\left[z_{1}[k], \ldots, z_{n-1}[k], z_{n+1}[k], \ldots, z_{n+m-1}[k]\right]^{T} .
\end{aligned}
$$

Then by some computations, we obtain the error systems as follows:

$$
\begin{aligned}
Z & {[k+1] } \\
& =\left[\begin{array}{cc}
A I_{n-1}-\alpha B C \widetilde{\mathscr{L}}_{1} & \alpha B C \widetilde{\Omega}_{1} \\
\alpha B C \widetilde{\Omega}_{2} & A I_{m-1}-\alpha B C \widetilde{\mathscr{L}}_{2}
\end{array}\right] Z[k] \\
& \triangleq F_{d} Z[k] \\
& =\left(A I_{n+m-2}+\alpha B C \widetilde{F}_{d}\right) Z[k]
\end{aligned}
$$

where

$\widetilde{F}_{d}=\left[\begin{array}{cc}-\widetilde{\mathscr{L}}_{1} & \widetilde{\Omega}_{1} \\ \widetilde{\Omega}_{2} & -\widetilde{\mathscr{L}}_{2}\end{array}\right]$,
$\widetilde{\mathscr{L}}_{1}=\left[\begin{array}{ccc}l_{11}-l_{n 1} & \cdots & l_{1(n-1)}-l_{n(n-1)} \\ \vdots & \cdots & \vdots \\ l_{(n-1) 1}-l_{n 1} & \cdots & l_{(n-1)(n-1)}-l_{n(n-1)}\end{array}\right]$,

$\widetilde{\mathscr{L}}_{2}=\left[\begin{array}{ccc}l_{(n+1) 1}-l_{(n+m) 1} & \cdots & l_{(n+1)(n+m-1)}-l_{(n+m)(n+m-1)} \\ \vdots & \cdots & \vdots \\ l_{(n+m-1) 1}-l_{(n+m) 1} & \cdots & l_{(n+m-1)(n+m-1)}-l_{(n+m)(n+m-1)}\end{array}\right]$,

$\widetilde{\Omega}_{1}=\left[\begin{array}{ccc}a_{1(n+1)}-a_{n(n+m)} & \cdots & a_{1(n+m-1)}-a_{n(n+m-1)} \\ \vdots & \cdots & \vdots \\ a_{(n-1)(n+1)}-a_{n(n+m)} & \cdots & a_{(n-1)(n+m-1)}-a_{n(n+m-1)}\end{array}\right]$,

$\widetilde{\Omega}_{2}=\left[\begin{array}{ccc}a_{(n+1) 1}-a_{(n+m) 1} & \cdots & a_{(n+1)(n-1)}-a_{(n+m)(n-1)} \\ \vdots & \cdots & \vdots \\ a_{(n+m-1) 1}-a_{(n+m) 1} & \cdots & a_{(n+m-1)(n-1)}-a_{(n+m)(n-1)}\end{array}\right]$.

Here we have used Assumption 1 and the property of Laplacian matrix.

Now the couple-group consensus problem of (7) has been converted into the stability problem of error system (16). We next give our main results.

Theorem 12. The multiagent system (7) can achieve couplegroup consensus asymptotically if and only if $\gamma$ and $(A, B, C)$ satisfy

$$
\begin{aligned}
& -\frac{A}{B C} \cos \left(\mu_{i}\right)-\frac{1}{B^{2} C^{2}\left|\mu_{i}\right|^{2}} \\
& \quad \times \sqrt{B^{2} C^{2}\left[A^{2} \operatorname{Re}^{2}\left(\mu_{i}\right)+\left(1-A^{2}\right)\left|\mu_{i}\right|^{2}\right]} \\
& \quad<\alpha
\end{aligned}
$$




$$
\begin{aligned}
< & -\frac{A}{B C} \cos \left(\mu_{i}\right)+\frac{1}{B^{2} C^{2}\left|\mu_{i}\right|^{2}} \\
& \times \sqrt{B^{2} C^{2}\left[A^{2} \operatorname{Re}^{2}\left(\mu_{i}\right)+\left(1-A^{2}\right)\left|\mu_{i}\right|^{2}\right]}, \\
& A^{2}\left(\operatorname{Re}^{2}\left(\mu_{i}\right)-\left|\mu_{i}\right|^{2}\right)+\left|\mu_{i}\right|^{2}>0,
\end{aligned}
$$

where $\mu_{i}(i=1, \ldots, n+m-2)$ is the ith eigenvalue of $\widetilde{F}_{c}$.

Proof. According to the aforementioned discussion, we know that the multiagent systems (7) can achieve couple-group consensus asymptotically if and only if the error system (16) is asymptotically stable. It follows from linear system theory [26] that system (16) which is asymptotically stable is equivalent to all eigenvalues of $F_{c}$ being within the unit circle. Denote the $i$ th eigenvalues of $\widetilde{F}_{d}$ by $\mu_{i}(i=1, \ldots, n+$ $m-2)$. Then $\rho\left(F_{d}\right)<1$ is equivalent to $\left(A+\alpha B C \operatorname{Re}\left(\mu_{i}\right)\right)^{2}+$ $\left(\alpha B C \operatorname{Im}\left(\mu_{i}\right)\right)^{2}<1$. That is,

$$
B^{2} C^{2}\left|\mu_{i}\right|^{2} \alpha^{2}+2 A B C \operatorname{Re}\left(\mu_{i}\right) \alpha+A^{2}-1<0 .
$$

By some computations, we know that if the conditions in (18) hold, then the inequality (19) is solvable. This completes the proof.

Remark 13. Theorem 12 provides a necessary and sufficient condition of couple-group consensus for multiagent system (7). According to linear system theory, we know that system (16) which is asymptotically stable is equivalent to that in which there exists a positive matrix $P$ such that $P-F_{d}^{T} P F_{d}>$ 0 . Hence, we can get another condition of couple-group consensus for multiagent systems (7).

Theorem 14. The multiagent system (7) can achieve couplegroup consensus asymptotically if and only if there exist positive definite matrices $P, Q$ and scalar $\gamma$ such that the following LMI

$$
\left[\begin{array}{cc}
P & F_{d}^{T} \\
F_{d} & Q
\end{array}\right]>\mathbf{0}
$$

holds with the constraint $P^{-1}=Q$. Here $F_{d}$ is defined in (16).

Proof. According to the discussion in Remark 13, and by using Schur complement lemma (Lemma 6) and letting $Q \triangleq$ $P^{-1}$, the proof can be obtained. This completes the proof.

Remark 15. Theorem 14 provides a necessary and sufficient condition of couple-group consensus for multiagent systems (7). We can get $\gamma$ by solving LMI in (20) with constrain $P^{-1}=$ $Q$. The cone complementarity linearization (CCL) method can be used to solve this problem $[19,29]$. We next summarize the algorithm as follows.

\section{Algorithm 16. Consider the following}

Step 1. Find a feasible point of LMI (20) $\gamma^{0}, P^{0}, Q^{0}$, set $k=0$. If there are none, exit.

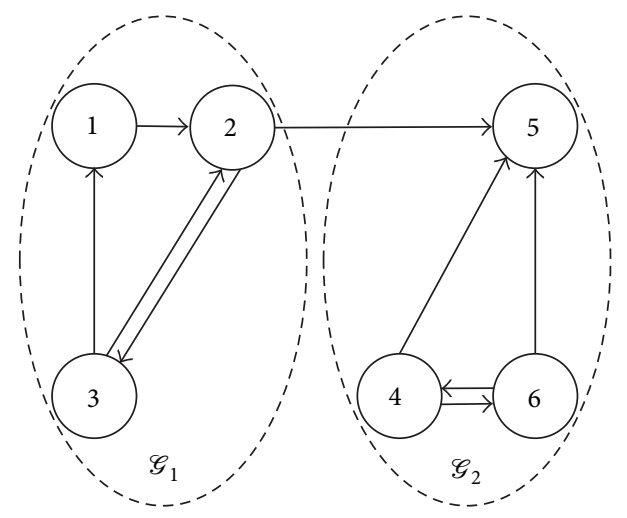

FIGURE 1: Topology $\mathscr{G}$.

Step 2. Find $\gamma^{k+1}, P^{k+1}, Q^{k+1}$ by solving the convex minimization problem

$$
t_{k}=\min \left\{\operatorname{tr}\left(P Q^{k}+Q P^{k}\right)\right\}
$$

s.t.

$$
\left[\begin{array}{cc}
P & F_{d}^{T} \\
F_{d} & Q
\end{array}\right]>\mathbf{0}, \quad\left[\begin{array}{cc}
P & I_{n+m-2} \\
I_{n+m-2} & Q
\end{array}\right] \succeq \mathbf{0} .
$$

Step 3. If $t_{k}=2(n+m-2)$, end this algorithm, and the feasible $\gamma$ is given by $\gamma=\gamma^{k+1}$. Otherwise, set $k=k+1$ and go to Step 2.

\section{Simulation Examples}

In this section, two examples will be given to show the usefulness of the theoretical results. For simplicity, we let $a_{i j}=1$ if $(i, j) \in \mathscr{E}$. On the other hand, we suppose that $a_{i j}$ takes values in a set $\{-1,0,1\}$ for $v_{i}, v_{j}$ belonging to different node sets, respectively.

Example 1. This example is for continuous-time multiagent systems. The interaction topology is as shown in Figure 1, which includes six nodes. It can be seen that the graph contains two subgraphs $\mathscr{G}_{1}$ and $\mathscr{G}_{2} \cdot\left(v_{1}, v_{2}, v_{3}\right) \in \mathscr{G}_{1}$, $\left(v_{4}, v_{5}, v_{6}\right) \in \mathscr{G}_{2}$. Each of them has a directed spanning tree. Let $A=0.3, B=0.8$, and $C=-0.9$. By solving the optimization problem in Algorithm 11, we obtain $\alpha=0.6304$ and

$$
Q=\left[\begin{array}{cccc}
32.3767 & -2.6303 & 0 & 0 \\
-2.6303 & 16.5170 & 0 & 0 \\
0 & 0 & 21.9891 & 0 \\
0 & 0 & 0 & 21.9891
\end{array}\right]
$$

The state trajectories of the agents are as shown in Figure 2. It can be seen that the agents belonging to $\mathscr{G}_{1}$ and $\mathscr{G}_{2}$ achieve two different consistent states, respectively.

Example 2. This example is for discrete-time multiagent systems. For simplicity, we suppose that the interaction topology is the same as that of continuous-time case, that is, $\mathscr{G}$. Let 


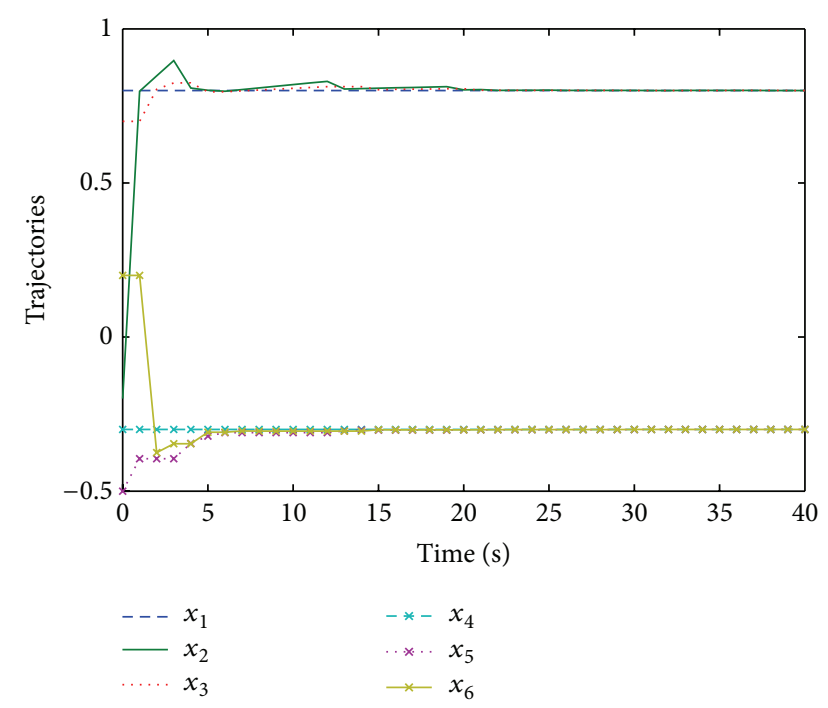

FIGURE 2: State trajectories for continuous-time case.

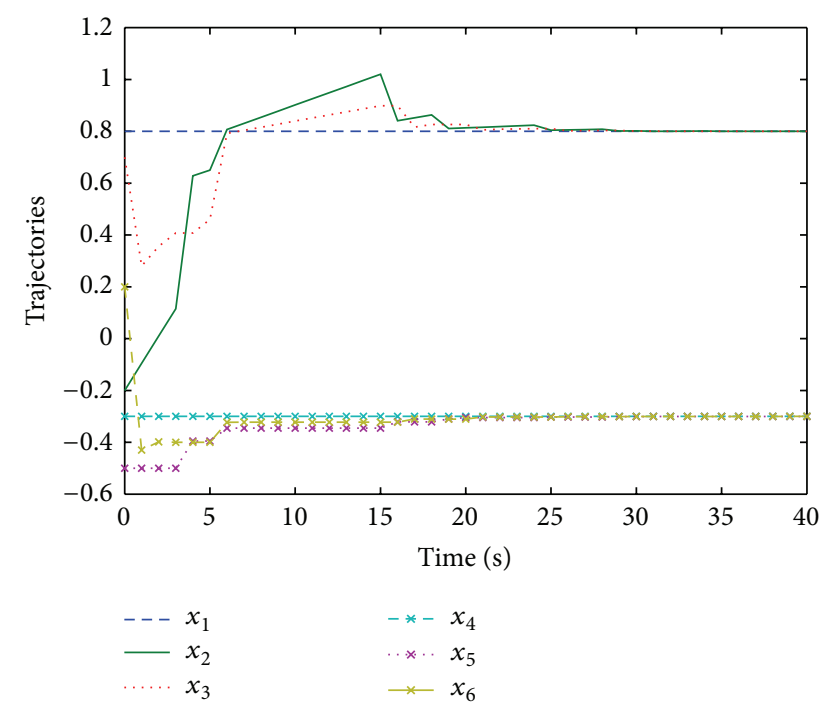

FIGURE 3: State trajectories for discrete-time case.

$A=0.4, B=0.7$, and $C=-0.8$. By solving the optimization problem in Algorithm 16, we obtain $\gamma=-0.2531$ and

$$
\begin{aligned}
P & =\left[\begin{array}{cccc}
1.7451 & -0.8085 & -1.2822 & 0.5011 \\
-0.8085 & 4.2494 & 6.6476 & -1.5457 \\
-1.2822 & 6.6476 & 13.4860 & -3.2780 \\
0.5011 & -1.5457 & -3.2780 & 1.9709
\end{array}\right], \\
Q & =\left[\begin{array}{cccc}
0.6426 & 0.1246 & -0.0273 & -0.1111 \\
0.1246 & 1.0569 & -0.5293 & -0.0832 \\
-0.0273 & -0.5293 & 0.3915 & 0.2430 \\
-0.1111 & -0.0832 & 0.2430 & 0.8746
\end{array}\right]
\end{aligned}
$$

Figure 3 shows the consensus results.

\section{Conclusion}

In this paper, we have studied the couple-group consensus problems for both continuous-time and discrete-time multiagent systems via output feedback control. By a system transformation, the consensus problems of multiagent systems have been converted into the stability problems of the error systems. Some necessary and sufficient conditions of couplegroup consensus for multiagent systems have been obtained. Two algorithms have been given to compute the allowable control gains. The effectiveness of the proposed results has been shown by the simulation examples.

\section{Conflict of Interests}

The authors declare that there is no conflict of interests regarding the publication of this paper.

\section{Acknowledgment}

The work was supported by the National Natural Science Foundation of China under Grants nos. 61203056, 61104007, 61203024,61273123 , and 61374061.

\section{References}

[1] W. Ren and R. W. Beard, Distributed Consensus in Multi-Vehicle Cooperative Control, Springer, Berlin, Germany, 2008.

[2] W. Ren and R. W. Beard, "Consensus seeking in multiagent systems under dynamically changing interaction topologies," IEEE Transactions on Automatic Control, vol. 50, no. 5, pp. 655661, 2005.

[3] W. Ren, "Multi-vehicle consensus with a time-varying reference state," Systems \& Control Letters, vol. 56, no. 7-8, pp. 474-483, 2007.

[4] J. Mei, W. Ren, and G. Ma, "Distributed coordinated tracking with a dynamic leader for multiple Euler-Lagrange systems," IEEE Transactions on Automatic Control, vol. 56, no. 6, pp. 14151421, 2011.

[5] J. Qin, H. Gao, and W. X. Zheng, "On average consensus in directed networks of agents with switching topology and time delay," International Journal of Systems Science, vol. 42, no. 12, pp. 1947-1956, 2011.

[6] H. Zhao, W. Ren, D. Yuan, and J. Chen, "Distributed discretetime coordinated tracking with Markovian switching topologies," Systems \& Control Letters, vol. 61, no. 7, pp. 766-772, 2012.

[7] D. Yuan, S. Xu, H. Zhao, and L. Rong, "Distributed dual averaging method for multi-agent optimization with quantized communication," Systems \& Control Letters, vol. 61, no. 11, pp. 10531061, 2012.

[8] G. Wen, Z. Duan, Z. Li, and G. Chen, "Consensus and its $L_{2}$ gain performance of multi-agent systems with intermittent information transmissions," International Journal of Control, vol. 85, no. 4, pp. 384-396, 2012.

[9] H. Liu, G. Xie, and L. Wang, "Containment of linear multiagent systems under general interaction topologies," Systems \& Control Letters, vol. 61, no. 4, pp. 528-534, 2012.

[10] W. Yu, G. Chen, M. Cao, and J. Kurths, "Second-Order consensus for multiagent systems with directed topologies and 
nonlinear dynamics," IEEE Transactions on Systems, Man, and Cybernetics, Part B: Cybernetics, vol. 40, no. 3, pp. 881-891, 2010.

[11] Y. C. Cao, W. W. Yu, W. Ren, and G. R. Chen, "An overview of recent progress in the study of distributed multi-agent coordination," IEEE Transactions on Industrial Informatics, vol. 9, no. 1, pp. 427-438, 2013.

[12] J. Yu and L. Wang, "Group consensus in multi-agent systems with switching topologies," in Proceedings of the 48th IEEE Conference on Decision and Control held jointly with 2009 28th Chinese Control Conference (CDC/CCC '09), pp. 2652-2657, December 2009.

[13] Y. Z. Feng, S. Y. Xu, and B. Y. Zhang, "Group consensus control for double-integrator dynamic multiagent systems with fixed communication topology," International Journal of Robust and Nonlinear Control, vol. 24, no. 3, pp. 532-547, 2014.

[14] Y. Feng, J. Lu, S. Xu, and Y. Zou, "Couple-group consensus for multi-agent networks of agents with discrete-time second-order dynamics," Journal of the Franklin Institute, vol. 350, no. 10, pp. 3277-3292, 2013.

[15] J. Yu, M. Yu, J. Hu, and B. Liu, "Group consensus in multi-agent systems with sampled data," in Proceedings of the 30th Chinese Control Conference, pp. 7168-7172, Xi'an, China, 2013.

[16] E. K. Boukas and Z. K. Liu, "Output feedback robust stabilization of jump linear system with mode-dependent time-delays," in Proceedings of the American Control Conference, pp. 46834688, Arlington, Va, USA, June 2001.

[17] F. Amato, M. Ariola, and C. Cosentino, "Finite-time stabilization via dynamic output feedback," Automatica, vol. 42, no. 2, pp. 337-342, 2006.

[18] J. C. Geromel, P. Colaneri, and P. Bolzern, "Dynamic output feedback control of switched linear systems," IEEE Transactions on Automatic Control, vol. 53, no. 3, pp. 720-733, 2008.

[19] J.-e. Feng, J. Lam, P. Li, and Z. Shu, "Decay rate constrained stabilization of positive systems using static output feedback," International Journal of Robust and Nonlinear Control, vol. 21, no. 1, pp. 44-54, 2011.

[20] C.-Q. Ma and J.-F. Zhang, "Necessary and sufficient conditions for consensusability of linear multi-agent systems," IEEE Transactions on Automatic Control, vol. 55, no. 5, pp. 1263-1268, 2010.

[21] H. Kim, H. Shim, and J. H. Seo, "Output consensus of heterogeneous uncertain linear multi-agent systems," IEEE Transactions on Automatic Control, vol. 56, no. 1, pp. 200-206, 2011.

[22] G. Zhai, S. Okuno, J. Imae, and T. Kobayashi, "A new consensus algorithm for multi-agent systems via dynamic output feedback control," in Proceedings of the IEEE International Symposlum on Intelligent Control, Part of 2009 IEEE Multi-conference on Systems and Control, pp. 890-895, Saint Petersburg, Russia, July 2009.

[23] K. You and L. Xie, "Coordination of discrete-time multi-agent systems via relative output feedback," International Journal of Robust and Nonlinear Control, vol. 21, no. 13, pp. 1587-1605, 2011.

[24] J. Yu and L. Wang, "Group consensus of multi-agent systems with undirected communication graphs," in Proceedings of the 7th Asian Control Conference (ASCC '09), pp. 105-110, Hong Kong, August 2009.

[25] A. Saberi, P. Sannuti, and B. M. Chen, $\mathscr{H}_{2}$-Optimal Control, Prentice-Hall, 1995.

[26] S. Boyd, L. El Ghaoui, E. Feron, and V. Balakrishnan, Linear Matrix Inequalities in System and Control Theory, vol. 15 of SIAM Studies in Applied Mathematics, SIAM, Philadelphia, Pa, USA, 1994.
[27] G. Zhai, M. Ikeda, and Y. Fujisaki, "Decentralized $H_{\infty}$ controller design: a matrix inequality approach using a homotopy method," Automatica, vol. 37, no. 4, pp. 565-572, 2001.

[28] S. L. Richter and R. A. DeCarlo, "Continuation methods: theory and applications," Institute of Electrical and Electronic Engineers. Transactions on Systems, Man, and Cybernetics, vol. 13, no. 4, pp. 459-464, 1983.

[29] L. El Ghaoui, F. Oustry, and M. AitRami, "A cone complementarity linearization algorithm for static output-feedback and related problems," IEEE Transactions on Automatic Control, vol. 42, no. 8, pp. 1171-1176, 1997. 


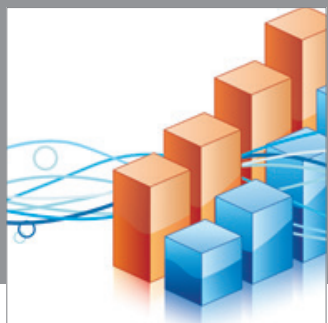

Advances in

Operations Research

mansans

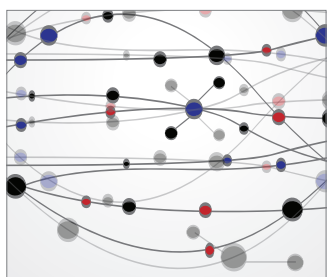

The Scientific World Journal
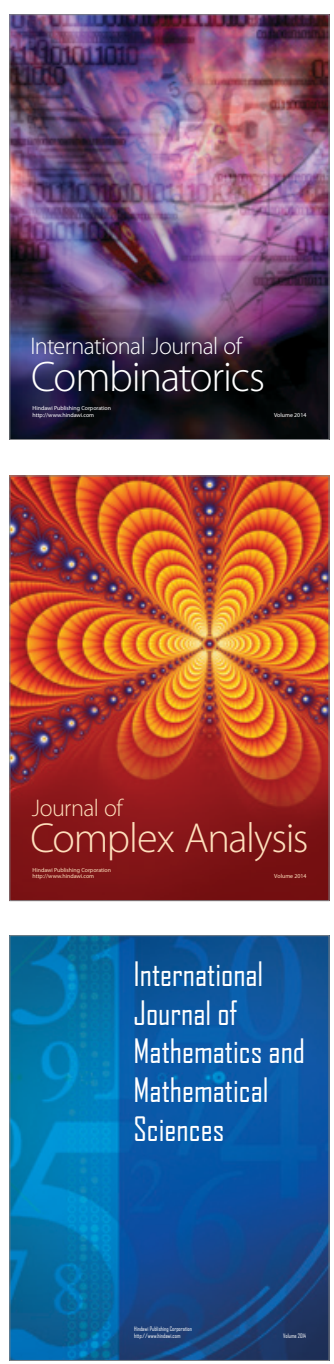
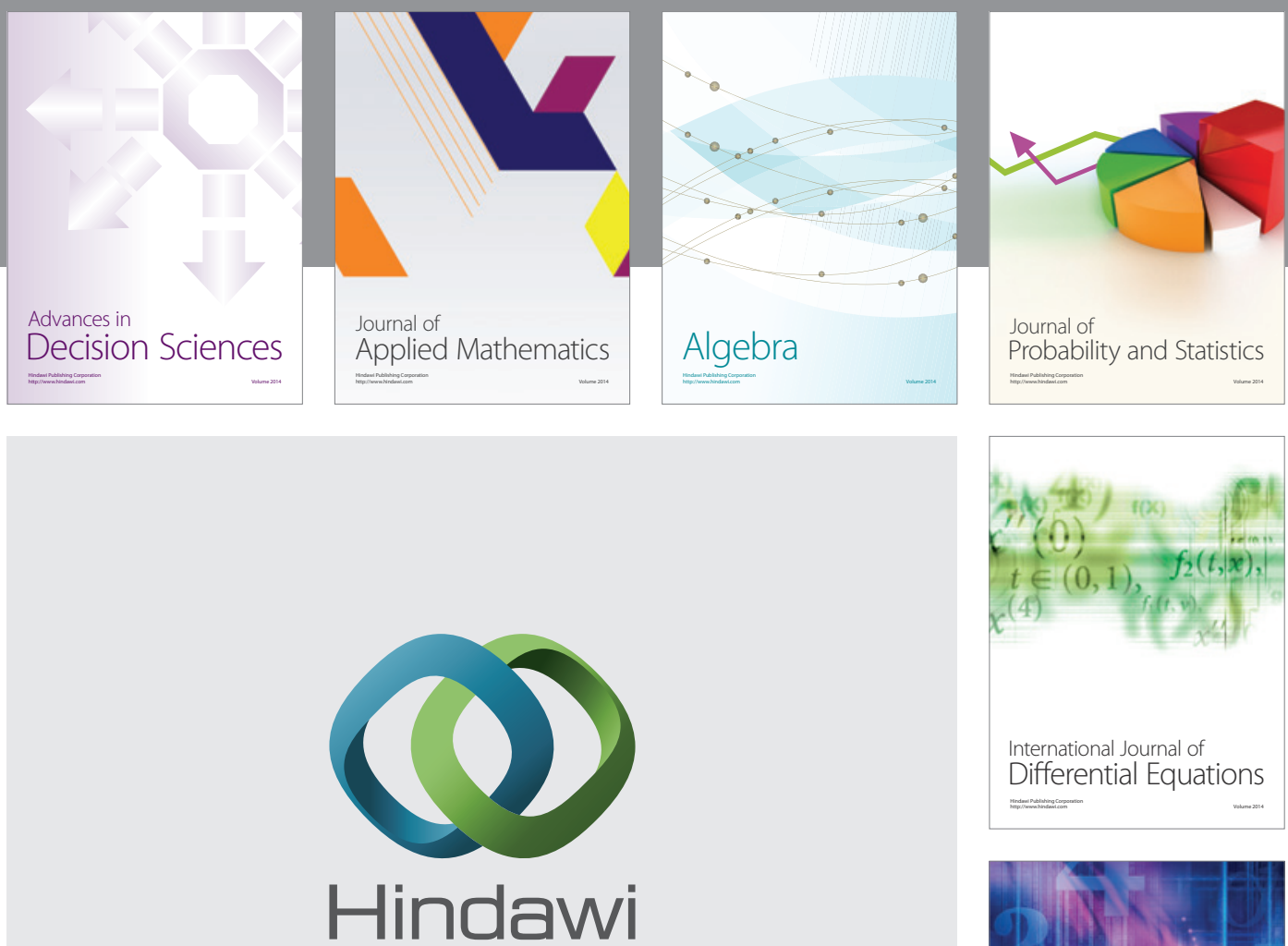

Submit your manuscripts at http://www.hindawi.com
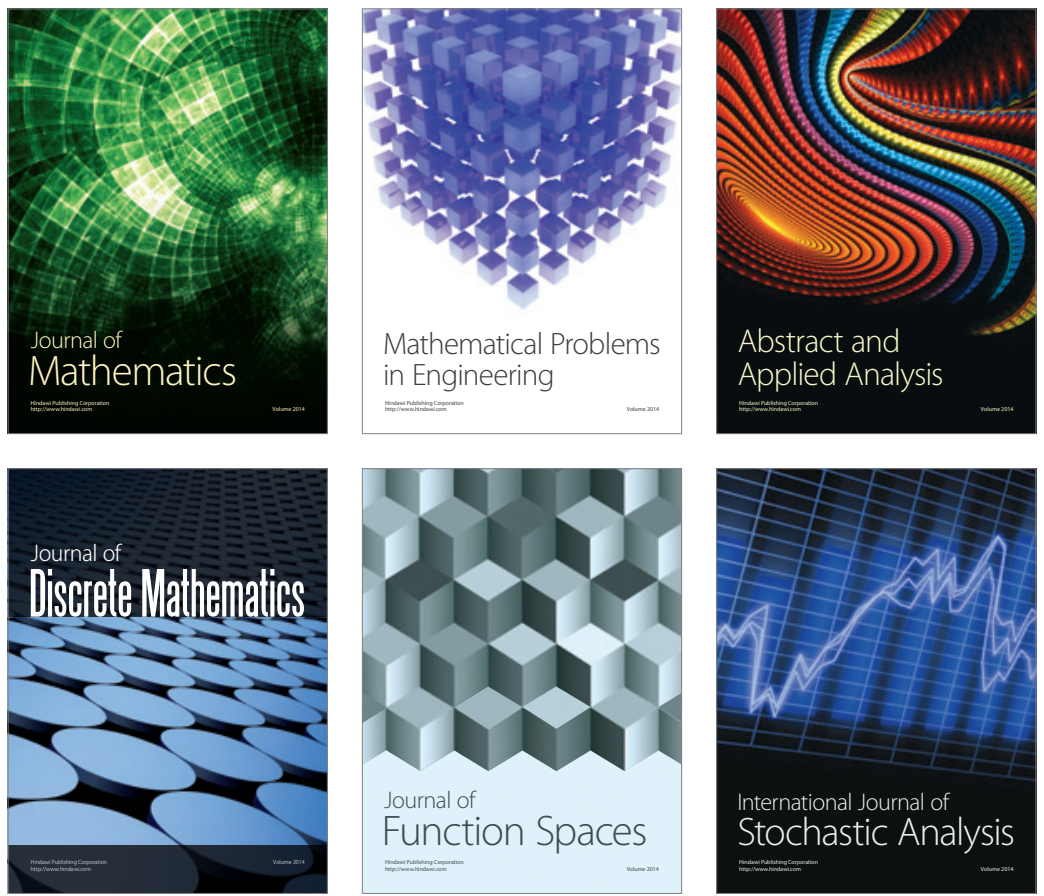

Journal of

Function Spaces

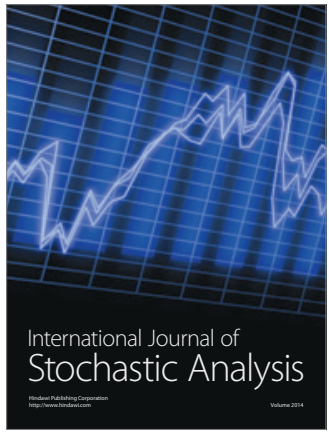

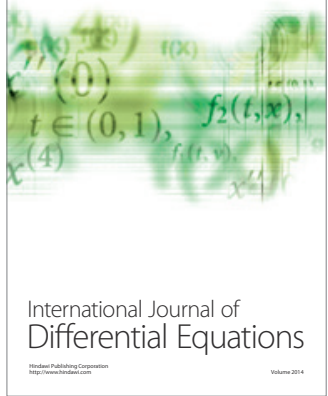
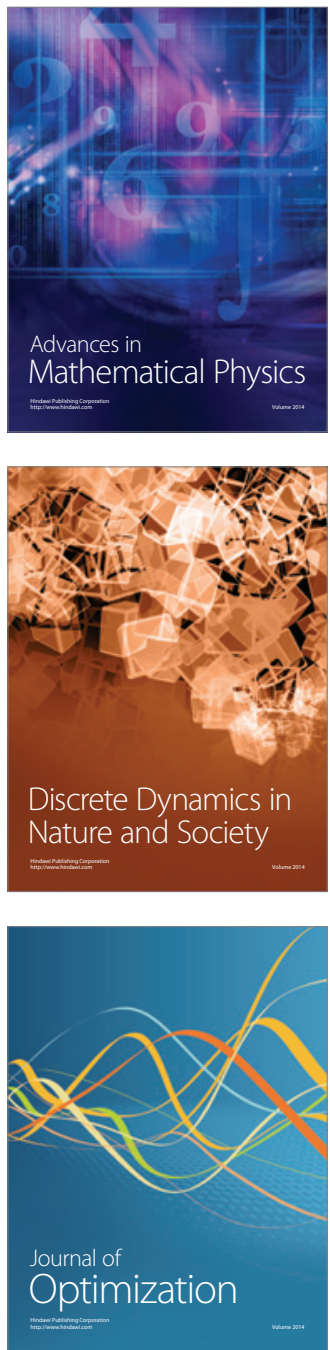